\title{
Optical Properties of Dense and Diluted Ensembles of Semiconductor Quantum Dots
}

\author{
M. V. Artemyev (a), A. I. Bibik (b), L. I. Gurinovich (b), S. V. Gaponenko (b), \\ H. JASCHINSKI (c), and U. WOGGON*) (c) \\ (a) Institute for Physico-Chemical Problems, Belarussian State University, Minsk 220080, \\ Belarus
}

(b) Institute of Molecular and Atomic Physics, National Academy of Sciences, Minsk 220072, Belarus

(c) Physics Department, University of Dortmund, Otto-Hahn-Str. 4, D-44221 Dortmund, Germany

(Received July 31, 2000; accepted October 2, 2000)

Subject classification: 73.21.La; 78.67.Hc; S8.12

\begin{abstract}
We examine collective effects in an ensemble of close-packed ultrasmall CdSe quantum dots by comparison of optical properties of dense and diluted thin film composites. Studies of absorption spectra, photoluminescence excitation spectra and electroabsorption of diluted and condensed structures along with numerical simulation confirmed that in a dense ensemble extended electron states occur by analogy with Anderson transitions in disordered solids. Therefore evolution from isolated quantum dots to quantum dot solids in case of strong confinement results in a drastic modification of optical and electronic properties.
\end{abstract}

1. Introduction During the last decades extensive studies have been performed of semiconductor nanocrystals in various matrices and solutions in the form of diluted and isolated particles and their principal features resulting from quantum confinement and interface effects were established [1,2]. It is reasonable to consider a transition from isolated and randomized nanocrystals to close-packed and spatially organized ensembles of nanocrystals as the next stage in these studies. With respect to nanocrystals self-organized to form a macroscopic colloidal crystal, a term "quantum dot solid" has been introduced [3] which implies a kind of condensed matter with spatial organization on a scale comparable to the electron de Broglie wavelength. The basic properties of dense quantum dot ensembles are expected to reproduce the features inherent in conventional solids, i.e. formation of energy bands in a perfect lattice and a coexistence of localized and delocalized electron states in a disordered quantum dot structure. By analogy with multiple quantum wells which form a planar superlattice one can consider quantum dot solid as three-dimensional superlattice formed by quantum dots. Recently we have reported [4] on systematic reversible modification of absorption spectrum of a quantum dot ensemble with dot concentration from a set of discrete subbands inherent in isolated nanocrystals to a smooth band-edge absorption similar to that of bulk semiconductors. The results were interpreted in terms of an evolution from individual (localized) to collective electron states delocalized within at least a finite number of nanocrystals. In the present contribution, we report on the further studies of dense quantum dot ensembles using thin films of small closepacked CdSe nanoparticles capped with organic group as a convenient model structure.

\footnotetext{
*) Supported by Volkswagen-Stiftung.
} 
2. Experimental We examine collective effects in an ensemble of close-packed CdSe quantum dots by comparison of optical properties of dense and diluted thin film composites. Ultrasmall CdSe quantum dots were synthesized as colloids of nanocrystals via wet chemical route in highly coordinating solvent pyridine. The nanocrystals of $1.6-1.8 \mathrm{~nm}$ in diameter, as determined by wide-angle X-ray diffraction are of narrow size distribution and contain surface stabilizing monolayer of 1-thioglycerol (FTIR data). Due to distinct ("magic") size of CdSe nanocrystals the absorption spectra of both diluted colloids and isolated quantum dots in polymethylmetacrylate (PMMA) matrix consist of a number of sharp bands in blue and near-UV regions (solid line in Fig. 1, left panel). The applied experimental techniques include optical absorption, photoluminescence emission, excitation and electroabsorption spectroscopies.

3. Results and Discussion Thin films formed by close-packed CdSe nanocrystals show systematic modification of the absorption spectrum compared to the diluted reference ensemble of the same nanoparticles dispersed in a polymer film. Unlike cluster-like spectrum inherent in the case of diluted nanoparticles, the absorption spectrum of close-packed nanoparticles is structureless and red-shifted. The effect occurs both at room and liquid helium temperatures (Fig. 1).

The observed modification of the absorption spectrum is attributed to the formation of collective electron states in a quantum dot ensemble. Further evidence of collective states in dense quantum dot structures can be found in photoluminescent properties. The photoluminescence spectra of both isolated and close-packed quantum dots possess a single broad band with large Stokes shift $(\approx 0.9 \mathrm{eV})$. Photoluminescence excitation spectra for isolated dots (Fig. 2) show the well-known shift of the peak with detection energy. Noteworthy, photoluminescence excitation spectra for close-packed dots are independent of detection energy. Inhomogeneous broadening of optical transitions in an ensemble of isolated dots due to their finite size vanishes with formation of collective electron states in a close-packed ensemble.
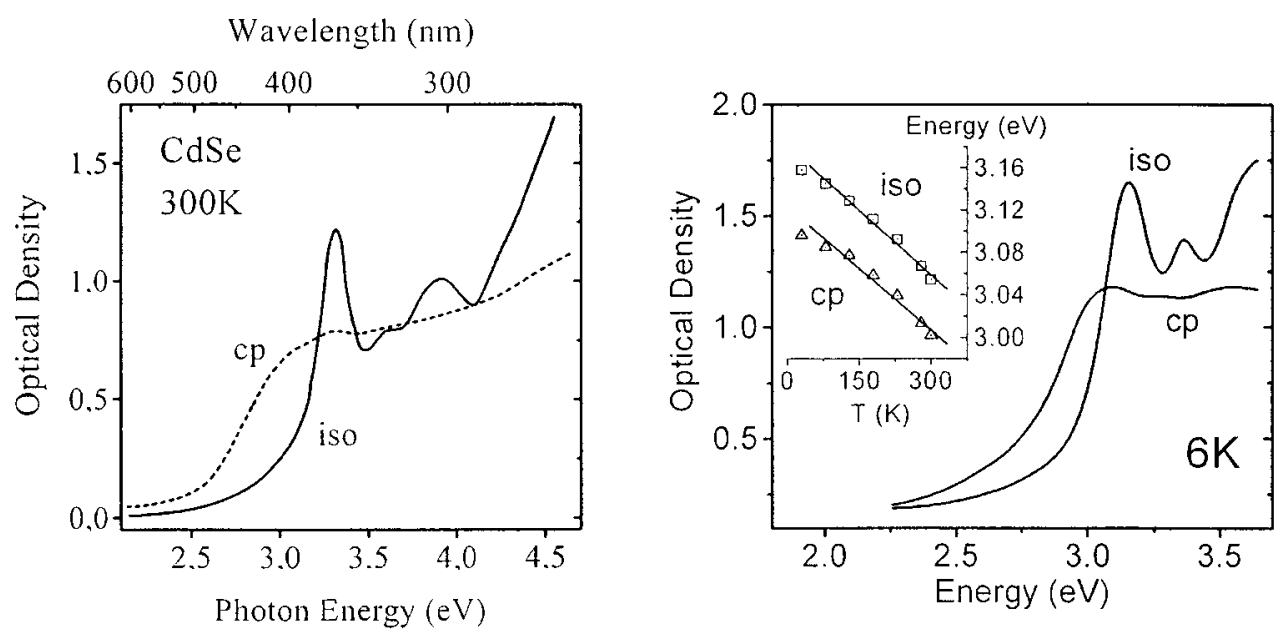

Fig. 1. Optical absorption spectra of isolated (iso) and close-packed (cp) ensembles of CdSe nanoparticles of average radius $1.6 \mathrm{~nm}$ at room (left panel) and cryogenic (right panel) temperatures. Insert shows the first peak position versus temperature 
To our knowledge, the problem of band structure calculations with respect to quantum dot solids has not been examined in detail to date. By analogy with atomic crystals, quantum dot solids are expected to possess electron and hole minibands in the case of an ideal superlattice of dots or coexisting localized and delocalized electron states separated by the mobility edge (Anderson transition) in the case of a dense but disordered quantum dot ensemble. The analogy with Anderson transition in disordered solids is more plausible for the structures investigated since no special measures have been made towards spatial ordering of nanoparticles.

To verify the above consideration, we performed numerical estimates for a system of $3375(15 \times 15 \times 15)$ wells randomly arranged within a fragment of a cubic lattice with a period $L$. The aim of calculations was to examine the number of delocalized states versus concentration of nanocrystals $C$, their radius $R$, and electron effective mass $m^{*}$. We consider a particle state as delocalized when the energy overlap integral

$$
I_{i j}=\int \Psi_{i}(r) H \Psi_{j}(r) \mathrm{d} r-\int \Psi_{i}\left(r^{\prime}\right) \Psi_{j}\left(r^{\prime}\right) \mathrm{d} r^{\prime} \int \Psi_{i}(r) H \Psi_{i}(r) \mathrm{d} r
$$

is higher than the difference of energy level shifts in the nearest-neighbor wells because of the influence of all other wells within the system [5]. Here $\Psi_{i}(r)$ and $\Psi_{j}(r)$ are electron wave functions in neighboring quantum wells with numbers $i, j$ and $H$ is the Hamiltonian of a system consisting of $i$ and $j$ quantum wells. Percentage of delocalized states is determined as ratio of quantum well pairs for which the delocalization condition holds to the total number of quantum well pairs. Random displacement of wells is described in terms of a deviation according to a Gaussian distribution of their coordinates with respect to nodes of a regular cubic lattice. Statistical analysis has been performed over 500 configurations of a quantum dot ensemble for every set of parameters $\left\{C, R, m^{*}\right\}$.

In Fig. 3 the number of delocalized electron states versus dot volume fraction is presented for various dot radii in the range of $0.5-2.0 \mathrm{~nm}$. In calculations the effective mass of electrons in bulk cadmium selenide was used. The results indicate the steady growth of delocalized electron states with increasing concentration of quantum dots. If the ratio of the dot radius and the average interdot distance $2 R / L$ is considered as a parameter, one can see that significant delocalization in case of larger dots occurs at $2 R / L \approx 1$, the vol-

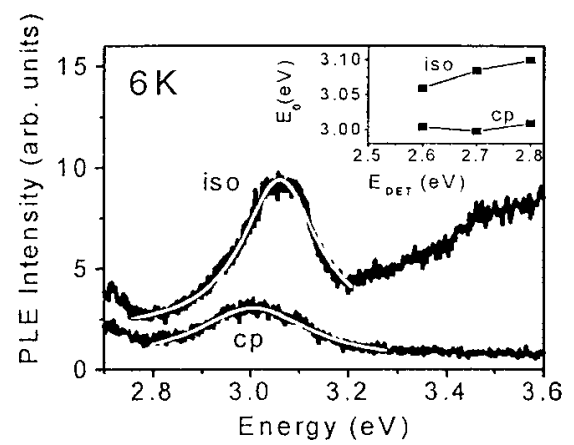

Fig. 2

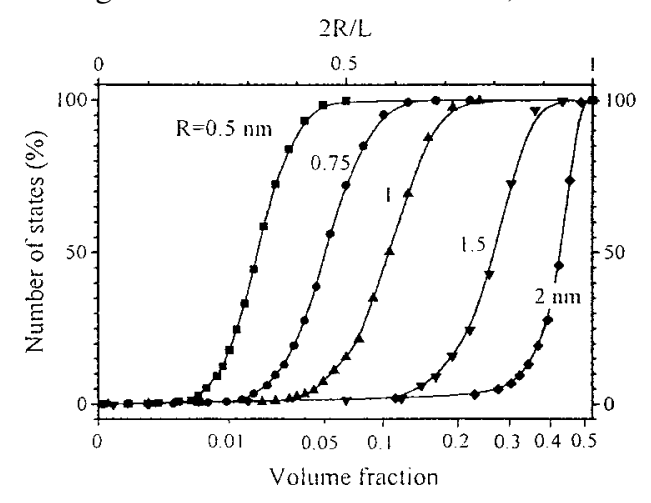

Fig. 3

Fig. 2. Photoluminescence excitation (PLE) spectra of close-packed (cp) and isolated (iso) CdSe quantum dots. Insert: PLE peak position versus detection energy

Fig. 3. Number of delocalized electron states versus volume fraction in an ensemble of quantum dots of radius $R$ randomly distributed within a simple cubic lattice of the period $L$ 
ume fraction being close to 0.52 that corresponds to close-packed arrangement of hard spheres in a simple cubic lattice. Therefore, modification of the absorption spectrum was not observed in the previous experiments [3] in which larger CdSe nanocrystals were examined. Taking into account presence of an organic shell, even close-packed ensembles of larger crystallites may not satisfy the delocalization condition. At the same time, for smaller dots a noticeable delocalization occurs at concentration far from close-packing, e. g. for $R=1 \mathrm{~nm}, 75 \%$ of electron states are delocalized at $2 R / L<0.6$. This corresponds to quantum dot volume fraction of 0.1 versus 0.52 relevant to maximal fraction of closepacked solid spheres in a simple cubic lattice. Therefore close-packed smaller quantum dots will reproduce, to a large extent, properties of atomic solids, including not only energy and charge transfer but a formation of collective energy states.

The coherency of extended states can be destroyed by means of external electric field due to field-induced band bending. This effect is known for planar semiconductor superlattices. Above a certain applied bias, the electric field dependent band bending results in a breaking of the minisubband structure and localization of electronic states within the wells. In the thin layers of close-packed nanocrystals studied here, the electron states are delocalized over several nanocrystals. With increasing electric field strength, the localization of electronic states in the single nanocrystals should be restored. This was observed recently in our experiments [6]. Unlike isolated nanoparticles, close-packed structures were found to show reversibly positive field-induced absorption changes which is indicative of vanishing of extended and restoration of individual confined electron states in the system under consideration.

4. Conclusions Systematic modifications of optical absorption and photoluminescence excitation spectra of a dense quantum dot ensemble compared to the reference diluted system are attributed to the formation of extended electron states in the quantum dot superstructure. The explanation is supported by numerical simulations of the Anderson transition in a dense quantum dot ensemble as well as by experiments on the external electric field effect which showed restoring of the original spectrum inherent in isolated nanoparticles when the field is applied to the condensed quantum dot system. These findings are believed to pose a number of issues related to electron properties of quantum dot superlattices like, e.g., electron band structure of a quantum dot supercrystal, renormalized electron and hole effective masses within a superstructure, modified electron-hole interaction (super-exciton) and others. One can foresee new prospects in synthesis of mesoscopic structures whose electronic and optical properties are controlled by three-dimensional confinement of electrons within every nanocrystal and by collective phenomena due to spatial organization of nanocrystals.

\section{References}

[1] U. Woggon, Optical Properties of Semiconductor Quantum Dots, Springer-Verlag, Berlin 1996.

[2] S. V. Gaponenko, Optical Properties of Semiconductor Nanocrystals, Cambridge University Press, Cambridge 1998.

[3] C. B. Murray, C. R. Kagan, and M. G. Bawendi, Science 270, 1335 (1995).

[4] M. V. Artemyev, A. I. BibiK, L. I. Gurinovich, S. V. Gaponenko, and U. Woggon, Phys. Rev. B 60, 1504 (1999).

[5] B. I. Shklovskir and A. L. Efros, Electron Properties of Doped Semiconductors, Nauka, Moscow 1979; Springer-Verlag, Berlin 1982.

[6] M. V. Artemyev, U. Woggon, H. Jaschinski, L. I. Gurinovich, and S. V. Gaponenko, J. Phys. Chem. B 104, 11617 (2000). 